\title{
Use of Antithrombin Concentrate for Acquired Antithrombin Deficiency in Acutely Unwell Children Receiving Unfractionated Heparin
}

\author{
Deepa R. Jayakody Arachchillage, MD, MRCP, FRCPath ${ }^{1,2,3}$ Mihaela Gaspar, BSc, MSc ${ }^{1}$ \\ Sukeshi Makhecha, BSc (Hons) Pharmacy ${ }^{1}$ Mike Laffan, DM, FRCP, FRCPath ${ }^{2,3}$
}

\footnotetext{
${ }^{1}$ Department of Haematology, Royal Brompton Hospital, London, United Kingdom

2 Department of Haematology, Imperial College Healthcare NHS Trust, Imperial College London, London, United Kingdom

${ }^{3}$ Centre for Haematology, Imperial College London, London, United Kingdom
}

Semin Thromb Hemost 2019;45:859-864.

Antithrombin (AT) is essential as a cofactor for unfractionated heparin (UFH) to deliver its anticoagulant effect. AT deficiency may result in inadequate anticoagulation with UFH at usual doses. Acquired AT deficiency is common in acutely unwell children due to either decreased production (especially in infants with liver immaturity) or increased consumption. It is known that children require higher doses of UFH than adults to achieve and maintain therapeutic levels of anticoagulation. ${ }^{1-3}$ In infants and children, the levels of AT are lower than in adults (may be as low as $10-30 \%$ ) and AT may be consumed in illness. ${ }^{3}$ In a study by Andrew et al, it was shown that children $<12$ months of age require a $50 \%$ higher UFH dose $(28 \mathrm{U} / \mathrm{kg} / \mathrm{h})$ compared with children $>12$ months of age $(20 \mathrm{U} / \mathrm{kg} / \mathrm{h})$ to achieve the same anticoagulant effect as measured by activated partial thromboplastin time (APTT). ${ }^{4}$ In another study, the median UFH dose required to achieve therapeutic anti-factor Xa (anti-Xa) levels in 83 infants was $33 \mathrm{U} / \mathrm{kg} / \mathrm{h}$ and $39 \%$ of infants required at least one dose of AT supplementation (50-200 IU/kg) to achieve therapeutic heparin anti-Xa levels. ${ }^{5}$ However, the UFH requirement may also be increased by nonspecific binding of UFH to acute phase reactants. Both reduced levels of AT and accelerated heparin clearance are barriers to effective anticoagulation in children.

Although the APTT is a cheap and easily available test that is commonly used for the monitoring of UFH, it may not provide an accurate measure of anticoagulant effect of heparin as a result of various confounding factors including both preanalytical and analytical variables. ${ }^{6}$ The anti-Xa assay is not as significantly affected by these confounding factors and so has been proposed as a better assay for monitoring UFH. Consequently, it is our standard practice

published online October 21, 2019
Issue Theme Recent Advances in Thrombosis and Hemostasis-Part V; Guest Editor: Sam Schulman, MD, PhD.

Address for correspondence Deepa R. Jayakody Arachchillage, MD, MRCP, FRCPath, Department of Haematology, Imperial College Healthcare NHS Trust, Imperial College London, Hammersmith Hospital, 4th Floor, Commonwealth Building, Du Cane Road, London W12 ONN (e-mail: d.arachchillage@imperial.ac.uk).

to use the anti-Xa level to monitor UFH rather than the APTT in both pediatric and adult patients. Based on comparisons using an anti-Xa chromogenic assay within our hospital, an APTT of 60 to 100 seconds (using SynthASil reagent on ACL TOP 500 [Instrumentation Laboratory]) corresponds to the recommended anti-Xa therapeutic range of 0.3 to $0.7 \mathrm{IU} / \mathrm{mL}$.

In this retrospective single-center observational study in a tertiary pediatric intensive care unit in United Kingdom, we set out to determine whether AT supplementation could elevate AT levels, achieve therapeutic anticoagulation based on heparin anti-Xa, and reduce UFH dosage. This was undertaken as a service evaluation project and approved by the Trust Clinical Effectiveness Unit. We studied children with acquired AT deficiency who were acutely unwell following acute thrombosis, cardiac surgery due to congenital heart disease, and those requiring extracorporeal membrane oxygenation (ECMO) for severe respiratory or cardiac failure or both. From January 2016 to March 2019, all patients $<16$ years who received AT concentrate (ATC) for acquired AT deficiency were included for the study.

Heparin anti-Xa activity was measured by chromogenic Liquid anti-Xa assay without exogenous AT, APTT was measured using SynthASil reagent, and AT concentration was measured using HemosIL Liquid Antithrombin (all from Werfen), on the same sample using an ACL TOP 500, before and 4 to 8 hours after administration of ATC. The Clauss fibrinogen level was also measured in the same sample using an FIB-C XL kit (Werfen) on the ACL TOP 500 analyzer.

The decision to administer ATC was based on the patient who required anticoagulation with UFH and failed to achieve therapeutic anticoagulation (measured by heparin anti-Xa) despite increasing the dose of UFH to $>40 \mathrm{U} / \mathrm{kg} / \mathrm{h}$ and was

Copyright (c 2019 by Thieme Medical Publishers, Inc., 333 Seventh Avenue, New York, NY 10001, USA. Tel: +1(212) 584-4662.
DOI https://doi.org/ 10.1055/s-0039-1700521. ISSN 0094-6176. 
found to have a low AT level. ATC was then administered to achieve an AT level of at least $0.5 \mathrm{IU} / \mathrm{mL}$. However, if a child needed UFH $>50 \mathrm{U} / \mathrm{kg} / \mathrm{h}$, even if the AT was $>0.5 \mathrm{IU} / \mathrm{mL}$, a dose of ATC was given to elevate the AT level to achieve an AT level $>0.7 \mathrm{IU} / \mathrm{mL}$. In addition, in children with suspected low AT levels based on clinical scenarios such as chylothorax or on ECMO, the AT level was measured to ensure it was not too low. In these children, if the AT levels were found to be very low (i.e., $<0.2 \mathrm{IU} / \mathrm{mL}$ ), AT replacement was given irrespective of the UFH dose to achieve an AT level $\geq 0.5 \mathrm{IU} / \mathrm{mL}$. Acute major bleeding and clinically relevant nonmajor bleeding were defined as per Subcommittee of the Scientific and Standardization Committee (SSC) of the International Society on Thrombosis and Haemostasis (ISTH). ${ }^{7}$

Results are reported as median or mean based on the distribution of results with range or standard deviation. Differences between pre- and post-ATC administration were analyzed using paired $t$-test. Spearman's correlation was used to evaluate the association between nonnormally distributed data. $p$-Values $<0.05$ were considered statistically significant in a two-sided test.

A total of 35 children received at least one dose of ATC during the study period. Median age was 6 (range: $0.33-$ 144) months and $45 \%$ were male. Clinical indications for anticoagulation were mechanical heart valve replacement (26\%), ECMO \pm thrombosis (23\%), venous and arterial thrombosis (20\%), chylothorax with thrombosis (17\%), and arterial thrombosis (14\%). Median AT level and heparin dose (ranges) prior to administration of ATC were $0.24 \mathrm{IU} / \mathrm{mL}$ (3-57) and $44.5 \mathrm{U} / \mathrm{kg} / \mathrm{h}$ (17-58), respectively. Median dose of ATC was 50 IU/kg (range: 20-92.6). Prior to administration of ATC, APTT was within the therapeutic range (60-100 seconds) in $26 \%$ (9/35) and supratherapeutic ( $>100$ seconds) in $60 \%(21 / 35)$, while only $40 \%$ (14/35) had heparin anti-Xa level within the therapeutic range $(0.3-0.7 \mathrm{U} / \mathrm{mL})$ with an overall median of $0.27 \mathrm{U} / \mathrm{mL}$ (range: $0.05-0.59$ ).

Within 4 to 6 hours following administration of ATC, there were significant increases in both AT (from $0.24 \mathrm{IU} / \mathrm{mL}$ [0.030.57 ] to $0.63 \mathrm{IU} / \mathrm{mL}$ [0.29-1.27], $p<0.0001$; - Fig. 1A) and heparin anti-Xa levels (from $0.27 \mathrm{U} / \mathrm{mL}$ [0.05-059] to $0.49 \mathrm{U} /$ $\mathrm{mL}$ [0.16-1.09], $p<0.0001$; - Fig. 1B), with $89 \%$ (31/35) of the children then achieving therapeutic heparin anti-Xa level. The correlation between AT dose and AT increment was moderate; $r=0.59$ (95\% confidence interval [CI]: 0.330.77 ), $p=0.0001$ (-Fig. 1C). APTT also significantly increased following ATC administration; median (range) APTT pre- and post-ATC administration was 112.8 seconds (30.3-300) and 148.3 seconds (29.8-312.2), $p=0.01$ (-Fig. 1D). However, only $11 / 35$ (32\%) of the children had an APTT concordant with their heparin anti-Xa level (10 had both therapeutic anti-Xa and APTT, while in one child both were subtherapeutic). - Fig. 2 demonstrates the APTT versus anti-Xa levels pre (A) and post (B) administration of ATC in 35 children.

Of the remainder, $21 / 35(60 \%)$ had therapeutic anti-Xa levels and supratherapeutic APTT and 3/35 (9\%) had subtherapeutic anti-Xa levels and supratherapeutic APTT. The increase in therapeutic anti-Xa assays was achieved despite a nonsignificant decrease in the dose of UFH (from $44.5 \mathrm{U} / \mathrm{kg} / \mathrm{h}$ [17-58] to $40 \mathrm{U} / \mathrm{kg} / \mathrm{h}[15-58], p=0.098)$. There was no difference in the Clauss fibrinogen level pre- and post-ATC administration; median: (range) $2.25 \mathrm{~g} / \mathrm{L}(1.3-5)$ and $2.35 \mathrm{~g} / \mathrm{L}(1.2-4.8)$, $p=0.12$. There were no significant adverse events such as major bleeding or allergic reactions. Overall, minor bleeding was observed in three $(8 \%)$ children ( - Table 1) with no difference in the pre- and postadministration of ATC. None of the children had allergic reactions to ATC. There was no recurrence of thrombotic events or new thrombosis in children on ECMO. A single dose of ATC was sufficient to maintain the therapeutic heparin anti-Xa level for at least 48 hours. After replacement of ATC, repeat AT level was tested only if the heparin anti-Xa level dropped below the therapeutic range with the established UFH dose over the previous 48 hours. Ten out of 35 children (29\%) received a second dose of ATC when their heparin levels dropped to $<0.3 \mathrm{U} / \mathrm{mL}$ with the currentdose UFH and if the AT level was $<0.40 \mathrm{IU} / \mathrm{mL}$. Four children received more than two doses of ATC because they were losing AT through the chylothorax. All children had AT levels normal for age once they recovered from acute illness at the time of discharge from hospital.

Thus, according to our evaluation, administration of ATC significantly increased both AT and heparin anti-Xa levels in keeping with other reported studies. ${ }^{3,8}$ Diaz et al studied 51 children with a median age of 3 months who received ATC during UFH therapy for acute thrombosis. Median AT level and UFH dose prior to administration of ATC were $0.61 \mathrm{IU} / \mathrm{mL}$ and $26 \mathrm{U} / \mathrm{kg} / \mathrm{h}$, respectively. Following a median ATC dose of $49.9 \mathrm{IU} / \mathrm{kg}$, both AT and anti-Xa level rose, although the majority of patients (86\%) did not undergo a change in UFH dose. ${ }^{8}$ In contrast, Ryerson et al found that high-dose ATC decreased UFH dose requirements. ${ }^{3}$ The median $(95 \% \mathrm{CI}$ ) doses of UFH prior to administration of ATC for children $\leq 12$ months of age and $>12$ months of age were $30.5 \mathrm{U} / \mathrm{kg} / \mathrm{h}$ (26.5-34.5) and $30.3 \mathrm{U} / \mathrm{kg} / \mathrm{h}(26.1-34.5)$, respectively, and fell to $18.9 \mathrm{U} / \mathrm{kg} / \mathrm{h}(16.8-20.9)$ and $17.7 \mathrm{U} / \mathrm{kg} / \mathrm{h}(15.0-20.4)$ following the ATC administration. Notably, in that published study $^{3}$ the mean dose of ATC was $241 \mathrm{IU} / \mathrm{kg}$ compared with approximately $50 \mathrm{IU} / \mathrm{kg}$ in our study and also in the report from Diaz and colleagues. ${ }^{8}$ With this higher dose of ATC, Ryerson et al achieved a mean AT level of $0.93 \mathrm{IU} / \mathrm{mL}$ from a baseline level of $0.40 \mathrm{IU} / \mathrm{mL}^{3}$ compared with $0.63 \mathrm{IU} / \mathrm{mL}$ from $0.24 \mathrm{IU} / \mathrm{mL}$ in our study and $0.79 \mathrm{IU} / \mathrm{mL}$ from $0.61 \mathrm{IU} / \mathrm{mL}$ in the study by Diaz et al. ${ }^{8}$

Following the higher dose of ATC administration in the Ryerson study, ${ }^{3}$ the mean anti-Xa increased from 0.23 to $0.41 \mathrm{U} / \mathrm{mL}$ and all children $\leq 12$ months of age achieved therapeutic heparin anti-Xa levels (mean $0.43 \mathrm{U} / \mathrm{mL}$ with 95\% CI of $0.33-0.53$ ). Although it was not clear how many children $>12$ months achieved therapeutic heparin anti-Xa, the mean was $0.35 \mathrm{U} / \mathrm{mL}$ (95\% CI: 0.26-0.44). The doses of ATC used in our study and in those of Diaz et $\mathrm{al}^{8}$ failed to achieve the age-normal AT concentration in many children (median: 0.63 and $0.79 \mathrm{IU} / \mathrm{mL}$, respectively). All children in the Ryerson study ${ }^{3}$ were on ECMO and $50 \%$ were $<3$ months of age and 26 were $<12$ months, so that the circuit loss and dilutional effect of ECMO with increased distribution volume may have helped avoid supra-normal AT levels. 

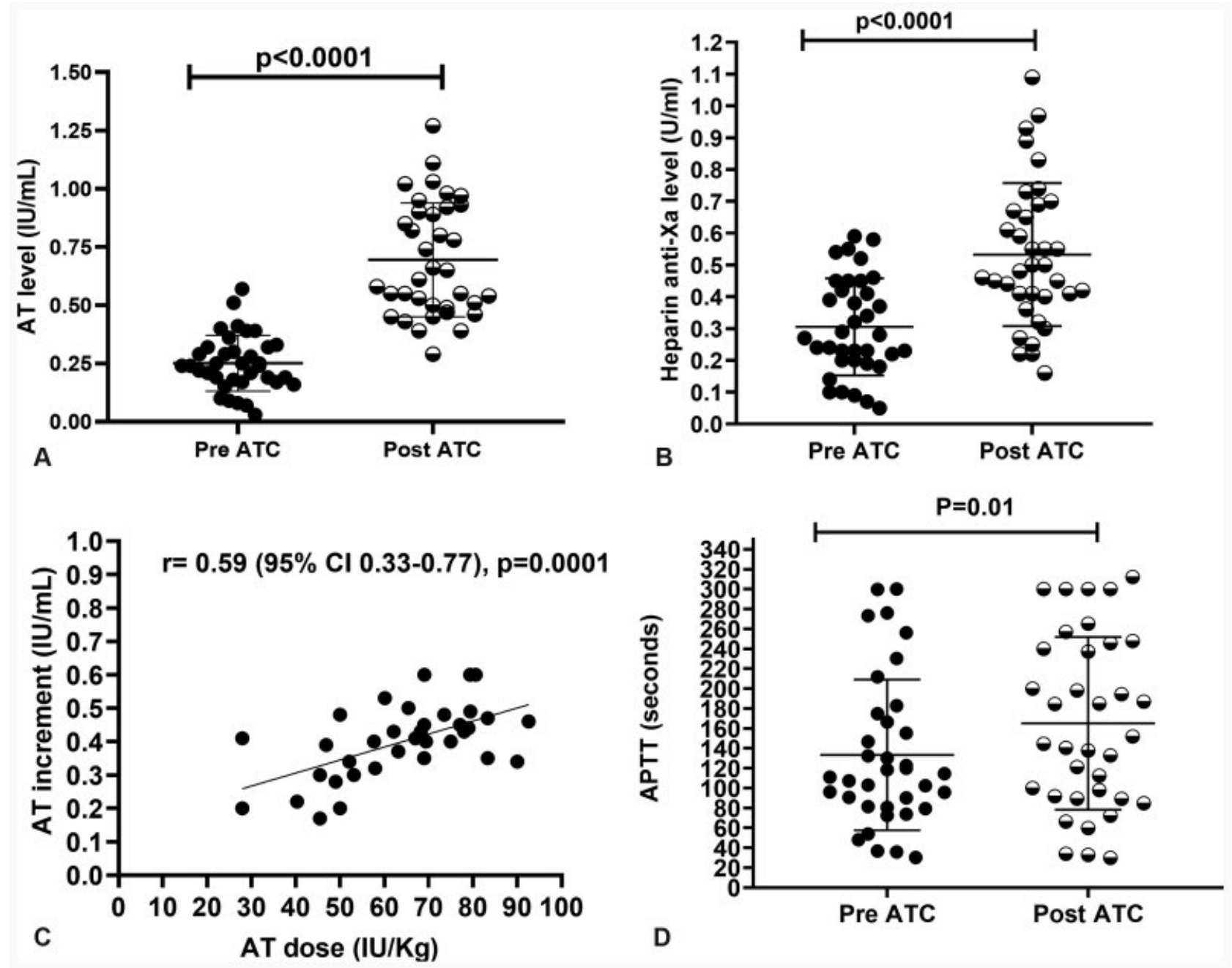

Fig. 1 (A) Antithrombin (AT) levels pre- and postadministration of antithrombin concentrate (ATC). (B) Heparin anti-Xa levels pre- and postadministration of ATC. (C) The correlation between AT dose and the increment of AT level from base line. (D) Activated partial thromboplastin time (APTT) pre- and postadministration of ATC. Error bars represent median \pm percentiles.
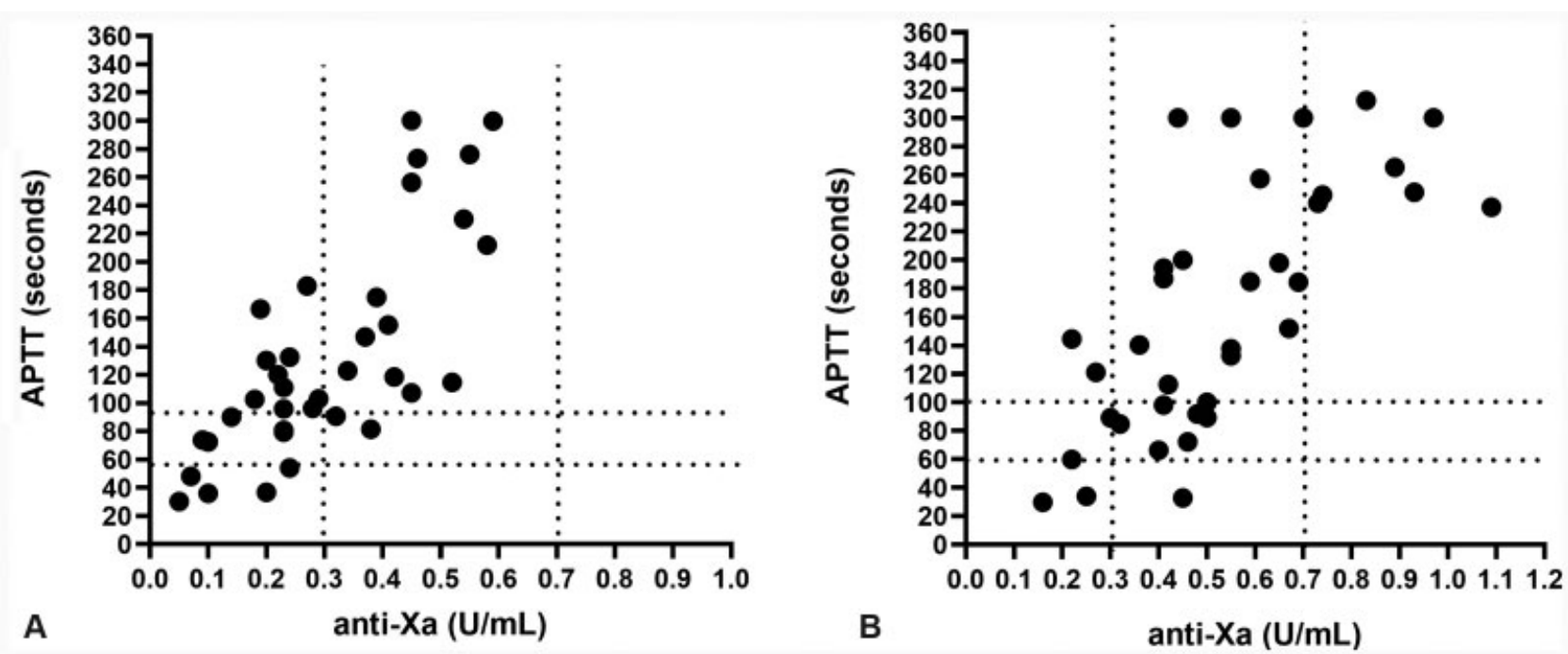

Fig. 2 Activated partial thromboplastin time (APTT) versus heparin anti-Xa levels pre (A) and post (B) administration of antithrombin concentrate (ATC). Dotted lines in each graph indicate the therapeutic range of APTT (60-100 seconds) and anti-Xa $(0.3-0.7 \mathrm{U} / \mathrm{mL})$ levels. 


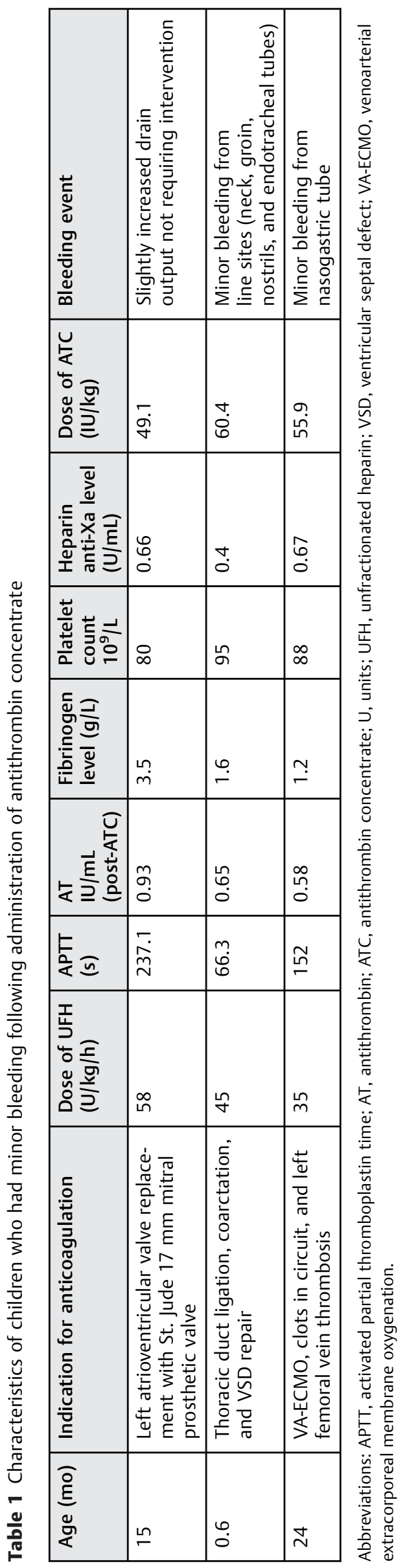

The significant UFH dose reduction identified in Ryerson et $\mathrm{al}^{3}$ suggests that AT can remain a limiting factor even after replacement.

ATC administration did not result in hemorrhagic complications or over-anticoagulation in the Ryerson et al study. ${ }^{3}$ Overall UFH doses across all age groups in our study pre (median: $44.5 \mathrm{U} / \mathrm{kg} / \mathrm{h}$ [range: $17-58$ ]) and post (median: $40 \mathrm{U} / \mathrm{kg} / \mathrm{h}$ [range: $15-58$ ]) were much higher than in the prior studies. ${ }^{3,8}$ We used a heparin anti-Xa assay that does not contain exogenous AT, which may give a more accurate estimation of anticoagulation in plasma but may not detect all the heparin present in that sample. Ryerson et $\mathrm{al}^{3}$ measured anti-Xa levels using an assay with no exogenous AT-the Rotachrom anti-Xa assay (Diagnostica Stago)-for part of the study (until November 16, 2009) and an assay with added exogenous AT-Biophen UFH anti-Xa assay (Hyphen BioMed)-during the rest of the study period (until May 2011). However, there was no direct comparison of the two methods as each assay was done on separate time points although authors mentioned that the anti-Xa results were similar in both assays. Diaz et $\mathrm{al}^{8}$ used STALiquid anti-Xa assay (Diagnostica Stago), which does not employ exogenous AT. Anti-Xa assay without exogenous AT cannot distinguish the difference between AT deficiency and insufficient heparin. However, the effect of ATC administration on heparin anti-Xa level without significant change on heparin dose indicates that the limiting factor in achieving adequate anti-Xa levels in our patients was low levels of AT. Although the heparin dose required is usually not $>50 \mathrm{U} / \mathrm{kg} / \mathrm{h}$ except in children with chylothorax, the very large doses of UFH used in our study could therefore be due to much lower AT levels (median: $0.24 \mathrm{IU} / \mathrm{mL}$ [range: 0.03-0.57]) in our cohort of children at baseline compared with $0.40 \mathrm{IU} / \mathrm{mL}$ in Ryerson et $\mathrm{al}^{3}$ and $0.61 \mathrm{IU} / \mathrm{mL}$ in Diaz et al. ${ }^{8}$

Although off-label use of ATC is increasing in both adult and pediatric practice, especially for ECMO, congenital AT deficiency is the only approved indication at present. The level of evidence base for use of ATC to support therapeutic anticoagulation with UFH is weak and mostly retrospective, including the current study. Conducting prospective randomized clinical studies in the pediatric population is extremely challenging and so accumulation of experience from large centers managing specific disease setting is important.

The major limitations of this study are small sample size, its retrospective design, and lack of a control group or prespecified ATC dosing regimen. However, despite being retrospective, almost all relevant clinical and laboratory data were available and patients represented a spectrum of indications to receive UFH.

In conclusion, AT deficiency, be it congenital or acquired, may limit anticoagulant therapy with UFH in sick children and that can be significantly improved after ATC replacement. Only $9 \%$ of children in our study had minor bleeding and none had recurrence of thrombosis. In children who fail to achieve therapeutic heparin anti-Xa levels despite using UFH $>40 \mathrm{U} / \mathrm{kg} / \mathrm{h}$ or those with chylothorax and associated thrombosis and unable to change to alternative 
anticoagulant, we believe it is reasonable to check AT levels and replace AT if the levels are $<0.40 \mathrm{IU} / \mathrm{mL}$.

\section{Authorship}

D.R.J.A. was involved in study concept and design, data collection, analysis, and interpretation of data, and prepared the first draft of the manuscript. M.G. and S.M. supported in data collection and revised the manuscript. M.L. was involved in interpretation of the data and revising the manuscript. All authors approved the final manuscript.

\section{Conflicts of Interest}

Authors state that they have no relevant conflict of interest.

\section{Acknowledgments}

We would like to thank Steve Owen and Maria Anievas for providing the accurate list of ATC issued from blood transfusion service, coagulation laboratory staff for performing the assays, and clinical staff at the pediatric intensive care unit for providing the clinical care.

\section{References}

1 Hirsh J, Warkentin TE, Shaughnessy SG, et al. Heparin and lowmolecular-weight heparin: mechanisms of action, pharmacoki- netics, dosing, monitoring, efficacy, and safety. Chest 2001;119(1, Suppl):64S-94S

2 Andrew M, Paes B, Milner R, et al. Development of the human coagulation system in the healthy premature infant. Blood 1988; 72(05):1651-1657

3 Ryerson LM, Bruce AK, Lequier L, Kuhle S, Massicotte MP, Bauman ME. Administration of antithrombin concentrate in infants and children on extracorporeal life support improves anticoagulation efficacy. ASAIO J 2014;60(05):559-563

4 Andrew M, Marzinotto V, Massicotte P, et al. Heparin therapy in pediatric patients: a prospective cohort study. Pediatr Res 1994; 35(01):78-83

5 Schechter T, Finkelstein Y, Ali M, et al. Unfractionated heparin dosing in young infants: clinical outcomes in a cohort monitored with anti-factor Xa levels. JThromb Haemost 2012;10(03): 368-374

6 Arachchillage DRJ, Kamani F, Deplano S, Banya W, Laffan M. Should we abandon the APTT for monitoring unfractionated heparin? Thromb Res 2017;157:157-161

7 Mitchell LG, Goldenberg NA, Male C, Kenet G, Monagle P, NowakGöttl U; Perinatal and Paediatric Haemostasis Subcommittee of the SSC of the ISTH. Definition of clinical efficacy and safety outcomes for clinical trials in deep venous thrombosis and pulmonary embolism in children. JThromb Haemost 2011;9 (09):1856-1858

8 Diaz R, Moffett BS, Karabinas S, Guffrey D, Mahoney DH Jr, Yee DL. Antithrombin concentrate use in children receiving unfractionated heparin for acute thrombosis. JPediatr 2015;167(03): 645-649 